\title{
Hybrid of Boyer Moore and Rule based System for Mobile Library Book Information
}

\author{
${ }^{1}$ Abd. Samad Hasan Basari, Noorrezam Yusop \\ ${ }^{2}$ Burairah Hussin, ${ }^{3}$ Abdul Samad Shibghatullah \\ Centre for Advanced Computing Technology, \\ Faculty of Information and Communication Technology, UTeM
}

\begin{abstract}
In this paper, a hybrid of Boyer Moore and Rule Based System are introduced for library book information on mobile application. A hybrid approach is employed as solution to replace the Digital Resource, Web based Software System and Catalogue. Quick Response (QR) Code is an improvement to replace the barcode and it can access any sensor on mobile application. The methodology of this paper is to develop Library Book Information to be more effective and by combining Boyer Moore String Matching and Rule Based System. From the phase of Boyer Moore and Rule Based System, these are greatly simplified this system by using QR Code to get data in a real time. The result in Boyer Moore and Rule Based System shows with highest priority or threshold (maximum) to minimum. It can be concluded that the hybrid of Boyer Moore and Rule Based System can effectively support the library information system.
\end{abstract}

\section{General Terms}

QR Code, Library Information System, Mobile Application

\section{Keywords}

Boyer Moore, String Matching, Rule Based System

\section{INTRODUCTION}

Library book information are typically identified during the early process whereby there are various of collections usually include such items as picture books, novels, non-fiction, nonprint materials, professional resources, and serials [1]. While inside of a library catalogues has consists of data about the author, title, subject, publisher, edition, place, year, language and ISBN of the book.

A hybrid approach involves the use of a combination of technology and non-technology solutions. Further, it will include a core infrastructure based on open standards to enable flexible development and modification, easy integration and interoperability, and intuitive access to services and information [2].

This research involves the use hybrid of Boyer Moore and Rule Based system as approach in library book information on mobile application. The Boyer Moore approach offers great flexibility because it obviates the need for time consuming index building and adapted to rapidly changing text bases. Moreover, Boyer Moore for String Matching uses two precomputed tables skip. It utilizes the occurrence heuristic of symbols in a pattern, and shift which utilizes the match heuristic of the pattern, for searching a string [3].

This has a motivation to support Rule Based System which is not only recognized for true/false but also an undetermined area. To do the rule based technique, one sample is taken as interference. The classical example in rule-based is to search book title. Furthermore, the rule-based approach can also prevent the system-based approach from instantaneous decision making on the large or small scale that may be pursued to answer upcoming concerns [4].

Quick Response (QR) Code that is two-dimensional code and an International Organization for Standardization (ISO) standard which can encode information such as text, URL or other data up to 7,089 numeric characters, 4,296 alphanumeric characters and 2,953 binary, has been used in many applications especially in mobile applications and the advantages to be used in Mobile Augment Reality applications [5]. QR codes can be found on store-front windows, magazines, newspapers, websites, posters, mass mailings, and billboards. Businesses display QR codes on advertisements to direct people to their websites. One study found over 14 million U.S. mobile users scanning QR codes during June 2011 especially in mobile applications [6]. The key reason is the demand from the user to provide access to information resources through their mobile is high that is one such technology which can cater to the user demand of providing access to resources through mobile.

Another work that promotes the Text search function techniques but has a different approach is available in most electronic document and as function matches string highlighted and there is a button to focus on to move on the next and previous occurrences. But a literal string matching has limitations. Issues of search queries will occur when readers who do not know exactly what they search for or how it might be expressed that match many extraneous passages or no passages at all. This happens if there is an advanced search functions is used to narrow down the search, and make people distressed [7]. The algorithm is reformulated by adding new feature to make it working as multi pattern string matching algorithm based on the hybrid recommender system [8]. This will boost the performance to overcome the weakness of the other string matching algorithms. However, this performance could affect a security issue in the modern network.

In this research, an enhancement work on a refined approach to support the process of accuracy and time taken in library book information on mobile application is proposed. This approach is an electronic library book information system on mobile application by using Boyer Moore and Rule Based derived from QR Code interaction pattern libraries.

\section{PROBLEM STATEMENT}

Library Book information is one of information or knowledge for student to get information by reading within educational or non-educational book. Most frequently student finds or tracks a book via computer that available in library. Nowadays libraries are having a paradigm shift towards web based eresources by utilizing an internet where the direction libraries 
operated. In certain libraries, the major collection would be in close access with a small collection and involve a staff to assist for the collection. The effectiveness of library depends on how they facilitate student/reader with desired bibliographic and web graphic resources. Libraries have to create environments that enable successful assessment and the implementation of results based on these assessments.

The standard existing application is used in catalogue and web base or intranet technology. This is the need for student to search manually in order to avoid of crowded from waiting other student while using the system.

A brief summary of the problem statements are

- Using Personal Computer (PC) machine is not efficient

- No additional features and information to student

- Use barcode can only keep small data

Moreover, in maintaining between traditional and electronic services, instructional information technology departments is monitoring the status and it is important to maintain technological currency in the face of decreasing resources, rising costs, and differing views about the funding priorities of the agency [1]. The implementation of using this database is important for information retrieval but a good computerized library system is essential and will help its users with quick and prompt services [9]. Based on these problems, this research is intended to propose hybrid approach for Boyer Moore String Matching and Rule Based System based on mobile application to solve current problem as described earlier.

\section{METHODOLOGY}

In order to design, develop and evaluate the proposed Library Book information on Mobile Application, the proof of concept application is developed using QR Code, Boyer Moore String Matching Algorithm and Rule-based Technique.

\subsection{Parameter Design}

\subsubsection{OR Code}

Figure 3.1 shows the design of QR Code in mobile application. It involves software as a middleware to connect the network to get the information from the client in a network. The mobile application camera will act as a sensor by scanning the QR Code to retrieve information from client on network. Then, the code will encode from QR Code in string or text file.

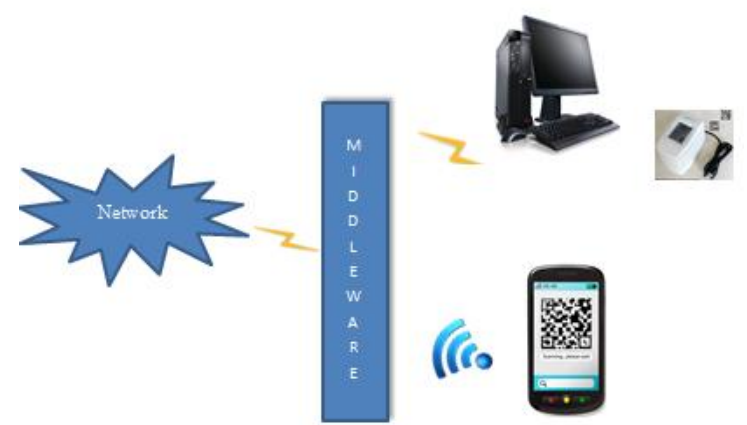

Figure 3.1 QR Code in Mobile Application and Personal Computer (PC)

\subsubsection{QR code and String Matching diagram}

Figure 3.2 shows that the design of QR Code and String Matching where the QR Code retrieved by sensor or camera to produce the data. The data or pattern will be analyzed in string matcher and at the same time it will point to every single character and a line of row in database to give the result in a list.

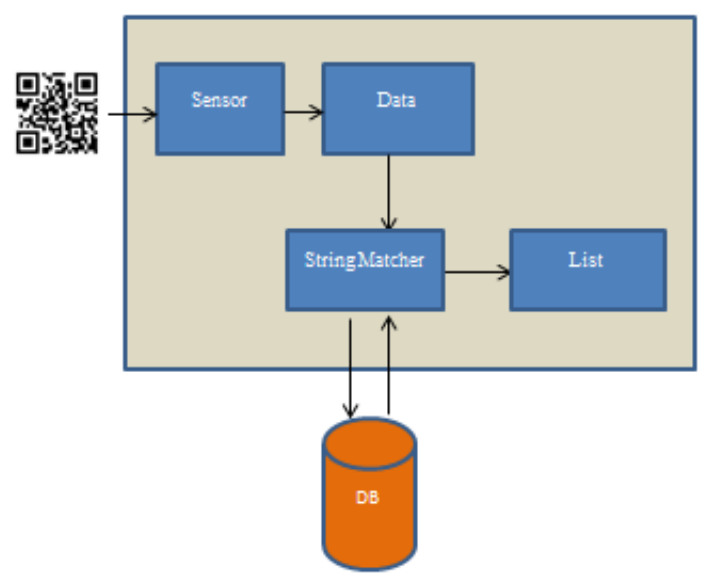

Figure 3.2 QR Code and String Matching Diagram

\subsubsection{Rule Based System}

Figure 3.3 shows the design of QR Code and string matching in the QR Code. The QR Code will be retrieved by Sensor or camera to produce the data. The data or pattern will be analyzed in string matcher and at the same time it will access at every single line of row in database to give the result in a rule based system. They will take part for achievement and store in a list.

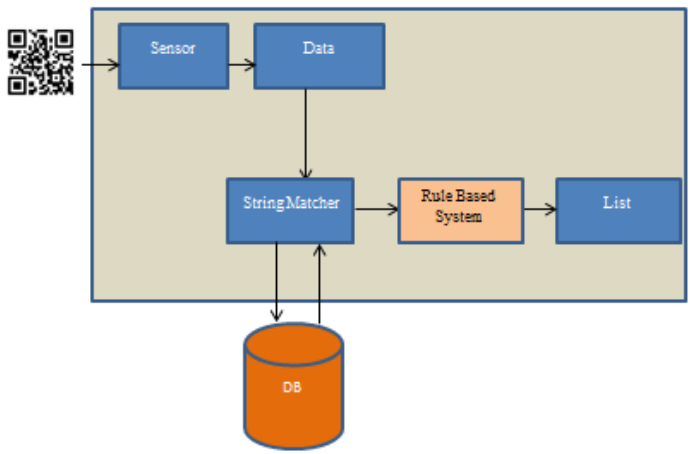

Figure 3.3 Hybrid Rule Based System and Boyer Moore Diagram

\subsection{Parameter Selection}

A parameter selection is taken into account for this approach as follows.

\subsubsection{Boyer Moore String Matching}

Boyer Moore String Matching aims to propose the measurement to get the result of the matched string. There are 2 parameters that will be measured according to the form of input namely Pattern $(\mathrm{P})$ and Text $(\mathrm{T})$. Sample of input and output is as follows:

Input: Pattern $\mathrm{P}[0 \ldots \mathrm{m}-1]$ and text $\mathrm{T}[0 \ldots \mathrm{n}-1]$.

Output: Index of left end first matching substring or -1 if there are no matches.

\subsubsection{Rule Based System}

In the Rule based system, the Degree of membership = No. of Pattern Match or No. of Text $=1$.

\subsection{Parameter Analysis}

There are some parameter analyses to clarify for the approach such as below. 


\subsubsection{Boyer Moore String Matching}

Boyer-Moore algorithm starts by aligning the pattern against the beginning characters of the text. If the first trial fails, it shifts the pattern to the right. The comparison within a trial that the algorithm does right to left, starting with the last character in the pattern.

$\mathrm{S}$

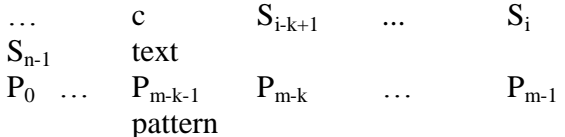

The algorithm for Boyer Moore String Algorithm and Pseudo code that proposed as below:

\section{Boyer-Moore Algorithm}

Step 1: Give pattern and alphabet used in both pattern and text. Construct bad symbol shift table as described earlier.

Step 2: Using pattern and construct the good-suffix table as describe earlier.

Step 3: Align pattern against beginning of text.

Step 4: Repeat the steps until either match substring is found or pattern reach last character of text. Start last character in the pattern. Then compare the corresponding character in pattern and text until either all $m$ character pairs are matched (then stop) or mismatch pair is encountered by $\mathrm{k}>0$ character pairs matched successfully. Retrieved entry $t_{1}(c)$ from $c$ column of bad-symbol table where $\mathrm{c}$ is a text mismatched character. If $\mathrm{k}>0$, retrieve correspond $\mathrm{d}_{2}$ entry from goodsuffix table. Shift pattern to right by number of position computed by formula

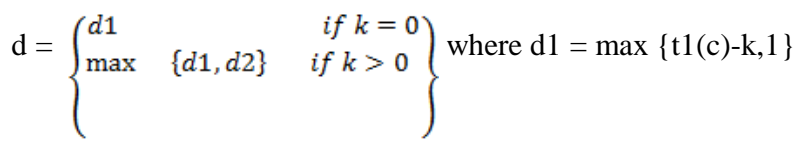

\section{Boyer Moore String Matching Pseudo code:}

$j \leftarrow m$;

$i \leftarrow m$;

while $(j>0$ and $i \leq n)$

$\{$ if $(x j=y i)$

$\{j \leftarrow j-1$;

$i \leftarrow i-1 ;\}$

else // a mismatch occurs

$\{i \leftarrow i+\max ($ skip [yi], shift $[j])$;

$j \leftarrow m ;\}$

l

if $(j<1) / / y(i+1, i+m)$ is a matching substring

$i \leftarrow i+1$;

else // no matching substring in string y

$i \leftarrow 0$;

return $i$;

\subsubsection{Rule Based System}

The total number of text that is needed to search must be identified. By looking the total number of text, the probability of each pattern based on pattern itself can be determined. The probability of pattern text must below or equal than the probability of Text. Figure 3.4 shows the rule based accuracy of text string matching.
Text = "This is a sample application modified by me" $=42$ characters include white space

Pattern $=$ "sample application detected" (18 characters was founds)

Figure 3.4 Rule Based Accuracy of Text String Matching

From that, green shaded is matched between the Text and Pattern about 18 characters was found. Thus, both accuracy and time taken of pattern in a list should be applied in rule based whereby it must be determined the attribute and it involve to If statement. The statement based on the result of the accuracy and time taken. It is assigned to the characteristic that want to define as the result

\subsection{Parameter Testing}

The parameter should be tested to prove the concepts of this research as below.

\subsubsection{Boyer Moore String Matching}

Figure 3.5 shows the Boyer Moore string matching part 1. The pattern's characters that are compared with their text counterparts are shown below.

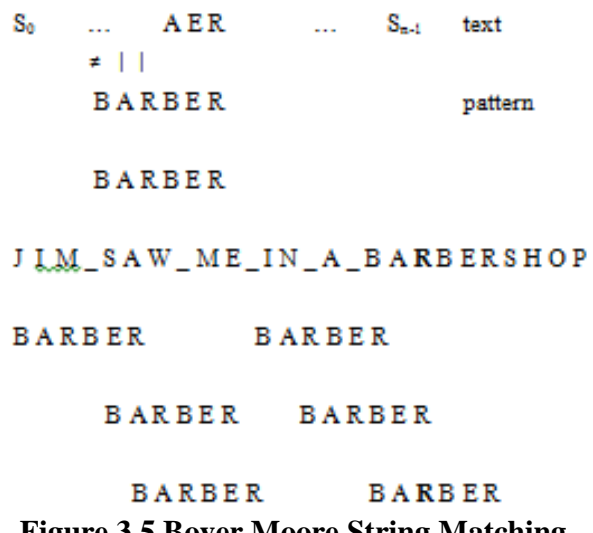

Figure 3.5 Boyer Moore String Matching

Figure 3.6 shows the second example of Boyer Moore string matching part 2. The pattern's characters that are compared with the text counterparts are in bold type.

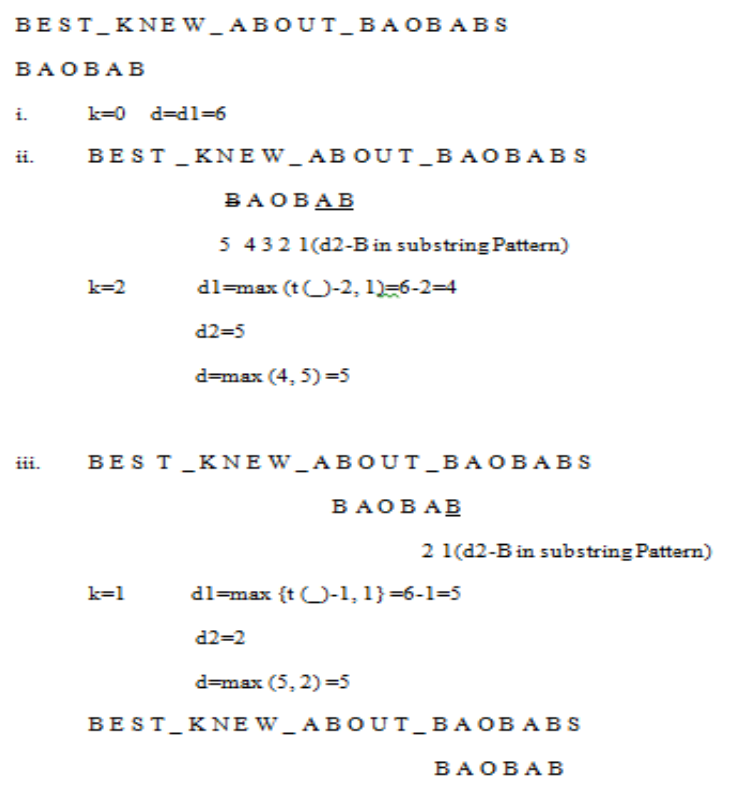

Figure 3.6 Boyer Moore String Matching 


\subsubsection{Rule Based System}

Table 3.1 shows the value of every attribute of accuracy, time taken and achievement. The attribute accuracy contains as low, medium or high to determine an accuracy of each string of text. While time taken as attribute when pattern of text is read for searching. It attributes value have a high, medium and low. The last attributes is an achievement mentioned the result between the accuracy and time taken which give score to top, average and low.

Table 3.1 Value of Every Attribute of Accuracy, Time Taken and Achievement

\begin{tabular}{|c|c|c|}
\hline ACCURACY & TIME TAKEN & ACHIEVEMENT \\
\hline LOW & HIGH & TOP \\
\hline LOW & HIGH & TOP \\
\hline LOW & HIGH & TOP \\
\hline LOW & MEDIUM & AVERAGE \\
\hline LOW & MEDIUM & AVERAGE \\
\hline LOW & SLOW & AVERAGE \\
\hline LOW & SLOW & DOWN \\
\hline LOW & SLOW & DOWN \\
\hline MEDIUM & HIGH & TOP \\
\hline MEDIUM & HIGH & TOP \\
\hline MEDIUM & HIGH & TOP \\
\hline MEDIUM & MEDIUM & AVERAGE \\
\hline MEDIUM & MEDIUM & AVERAGE \\
\hline MEDIUM & MEDIUM & AVERAGE \\
\hline MEDIUM & SLOW & AVERAGE \\
\hline MEDIUM & SLOW & AVERAGE \\
\hline MEDIUM & SLOW & AVERAGE \\
\hline HIGH & HIGH & TOP \\
\hline HIGH & HIGH & TOP \\
\hline HIGH & HIGH & TOP \\
\hline HIGH & MEDIUM & AVERAGE \\
\hline HIGH & MEDIUM & AVERAGE \\
\hline HIGH & MEDIUM & AVERAGE \\
\hline HIGH & SLOW & AVERAGE \\
\hline HIGH & SLOW & AVERAGE \\
\hline HIGH & SLOW & AVERAGE \\
\hline
\end{tabular}

The chapter explained each of the four parameter phases including parameter design, parameter selection, analysis and testing. This parameter explains and describes the detail design and process used during design and implementation to produce expected outcome or result. Moreover, this technique is pretested and examined the utilities before suggesting as methods of measurement. The clear approaches give confidence to achieve the objective and from the result to analyze the data.

\section{IMPLEMENTATION}

The implementation process starts from the design of MySQL server database, algorithm writing code for Boyer Moore and rule based system and application interface design. The details mentioned as follow.

\subsection{Design of MySQL Server Database}

Figure 4.1 shows the database design in MySQL Server. There are 11 attribute involves in this situation. The attribute of product ID is 'pid' as Primary Key to define that information in the table is a unique ID and cannot be duplicated and isbncode is the product code for the book. Other attribute involves such as titlebook, author, publisher, price, description, create_at, update_at, QR_ImageFile and Book_ImageFile as shown in Figure 4.1.

\begin{tabular}{|c|c|c|c|c|c|}
\hline \# Name & Type & Collation & Attributes Null & I Default Extra & Action \\
\hline 1 idd & $\operatorname{int}(5)$ & & No & None AUTO_NCREMENT & 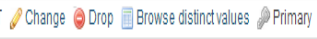 \\
\hline (1) 2 ishncode & text & lain1_swedish_o & No & None & 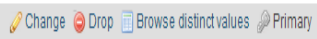 \\
\hline 3 tittebook & text & laiin__swedish_cl & No & None & Change Crop Browsedistincivalues Primary \\
\hline (1) 4 author & text & laiin1_swedish_c & No & None & $\nabla$ Change $\theta$ Crop $\square$ Erowse distinct values $P$ Pimary \\
\hline (1) 5 publisher & $\operatorname{tant}$ & laiin__swedish_c & No & None & Change (Crop G Erowse distincivalues Primary \\
\hline$\square$. price & couble & & No & None & Change 0 Crop $\square$ Browse distinct values Primayy \\
\hline 7 description & text & lain1_swedish_c & No & None & Change Crop Browsedistinctualues Plimary \\
\hline [ 8 created_at & cate & & No & None & Change 0 Crop $\square$ Browsedistincuvalues Primary \\
\hline (1) 9 updated_at & date & & No & None & 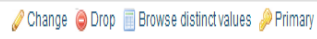 \\
\hline (1) 10 QR_Imagefile & text & lain1__wedish_c & No & None & Change 0 Crop $]$ Browse distinctualues Primary \\
\hline [] 11 Book_Imagefle & & laiin1_swedish_c & No & None & Change Crop Browsedistinctualues Primary \\
\hline
\end{tabular}

Figure 4.1 Database Design for Table Book

\subsubsection{Algorithm Writing Code}

The algorithm to be executed involves in this development or implementation research as below:

The following algorithm shows the accuracy and time taken in Figure 4.2(a) and Figure 4.2(b) of Boyer Moore string matching.

\section{Boyer Moore string matching algorithm code:}

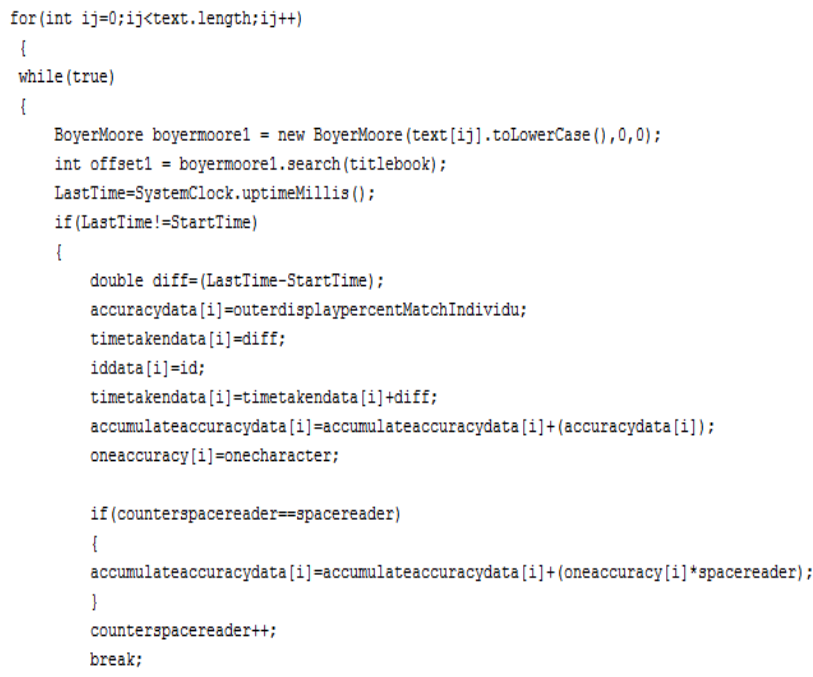

Figure 4.2(a) Algorithm to Calculate Accuracy and Time Taken 


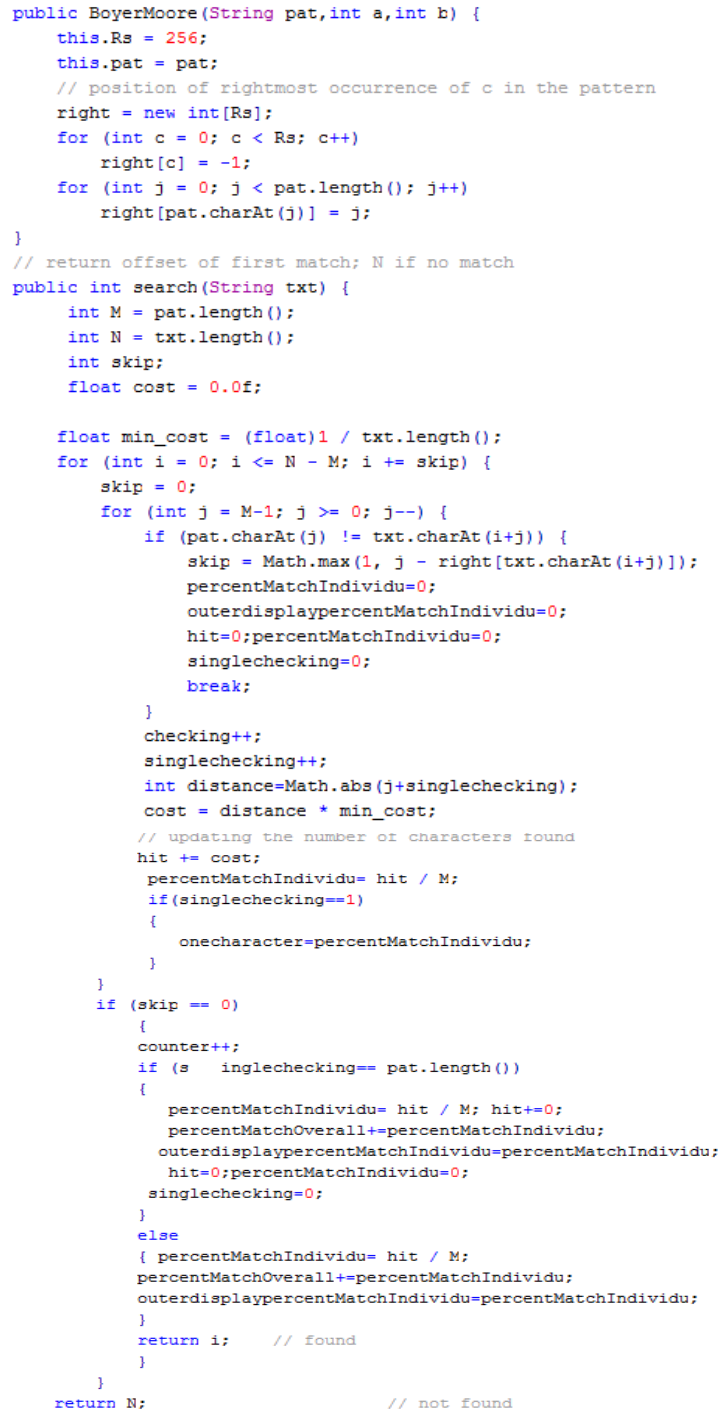

Figure 4.2(b) Algorithm Code for Boyer Moore

\section{Rule based system algorithm code}

Figure 4.3 show that there are 2 input attributes and 1 output attribute in this research for rule based system. Accuracy and time taken will be used as input to give an output product.

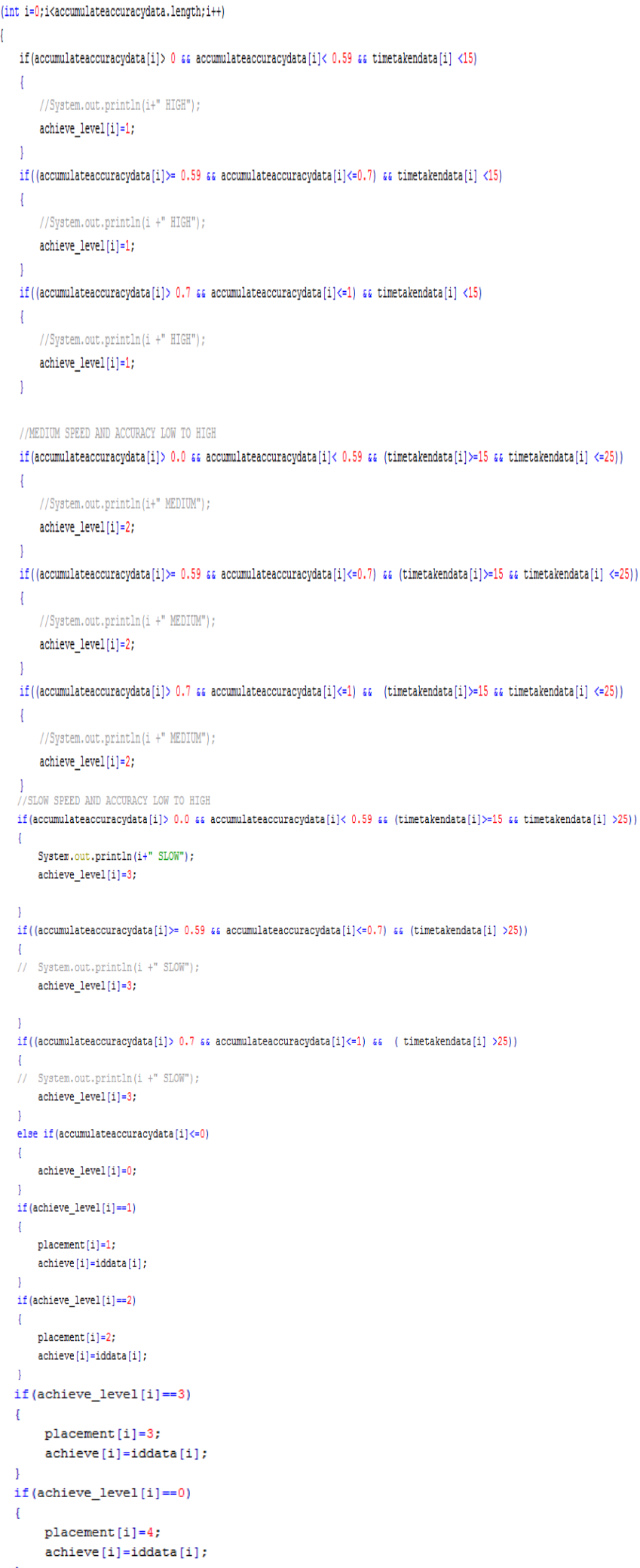

Figure 4.3 Algorithm Code for Rule Based System

4.1.2 Interface Design of Application

Figure 4.4 shows the dashboard application for book information during executing this system. From this dashboard menu, this application is tested by using QR Code with Boyer Moore and rule based as experimented below. 


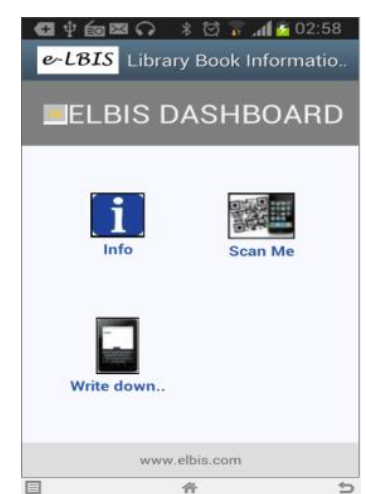

Figure 4.4 Dashboard Application and Scanned Book Interface

Figure 4.5 shows the Book with QR code that is scanned by camera. The information of book is produced based on database from MySQL Server. The product code of ISBN is 20001335 for the experiment below.

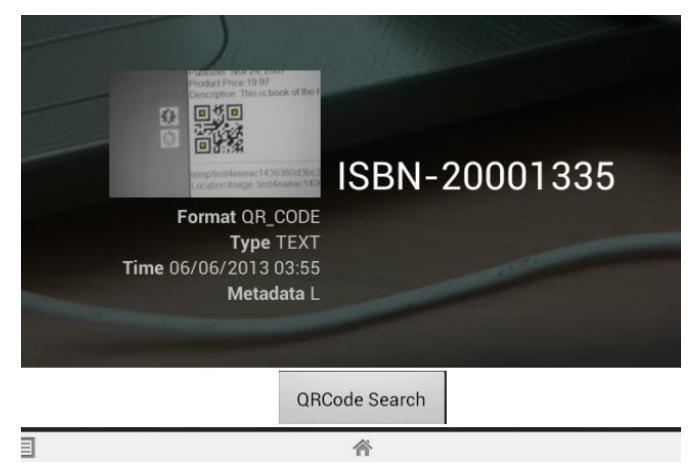

Figure 4.5 Scanned QR Code by Camera Sensor based on Mobile Application

Figure 4.6 shows the book information that has been encoded with QR code. The information of book is produced based on database from MySQL Server itself. The product code of ISBN is 20001335.

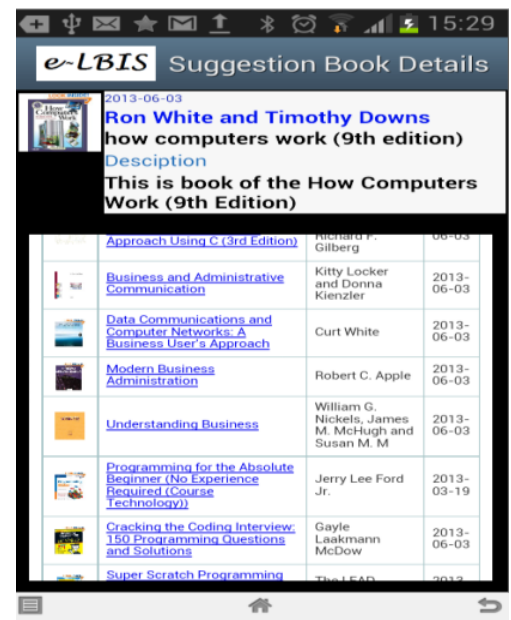

Figure 4.6 Pattern Information of Book with Other Suggested Book

Figure 4.7 shows the detail information of the book when users click the related book title. Based on the information, user can get the information via real time mobile application.

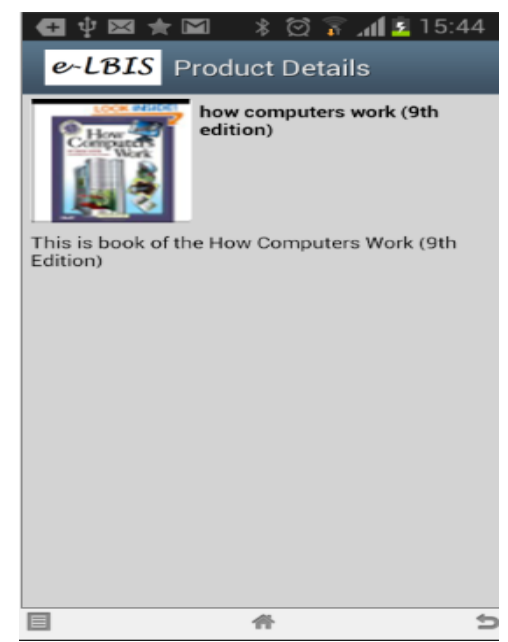

Figure 4.7 Details of Book Information

\subsection{Experimental Tasks}

These experiments used 30 samples of book that store in the MySQL Server. QR Code is scanned which is placed on the back of the book. Finally, from this chapter, a clear view on the design and implementation are explained. The design includes the database design, the writing code from algorithm and application design. The database design contains the attribute that store the collection of metadata. The translation code from algorithm of Boyer Moore and rule based system are required where this involve some functional and process for accuracy, time taken and achievement. The selected parameter is tested to evaluate the research after using Boyer Moore and rule based technique. Based on the methodology explains in Chapter 3, the information to design and implement this research is discussed. Then, the result of the test is based on experiment that is explained and discussed in Chapter 5.

\section{RESULTS}

This research have designed and developed a hybrid of Boyer Moore and rule based system for mobile library book information. However, some significant improvements and contributions are made over such predecessor systems. The phase of Barcode and QR Code have shown a greatly simplified in this system and QR Code is better in current nowadays technology. The android application is very effective when using Boyer Moore and rule based system because of the accuracy of pattern text. This kept and counted every single keywords searching until the end of the text. There is no loss accuracy and time taken because each of the text can be read. The result has been proven by Boyer Moore engine for each single entry row of table on database.

\subsection{Result and Discussion}

Table 5.1 shows the information by using Boyer Moore and rule based system. The Id 16, ISBN Code, ISBN-20001335 with the title book, "how computer work ( $9^{\text {th }}$ Edition)" is used as a pattern that is used to be searched in book collection from electronic library database. During this process, the accuracy has matched the probability rules of 1.0 or $100 \%$ totally matched. The time taken during searching process is 2 milliseconds. It means that Boyer Moore starts from Id 1 until the last length of Id. In these 30 samples, the accuracy has matched at row number Id 16 as shaded on Table 5.1. The time taken is counted at start entry row until the next row to be searched based on the pattern. 
There are 7 samples that applicable to reach the accuracy of the pattern. Other 23 samples test is tendency to low accuracy. It can be seen that the interval time read in sample 1 to sample 18 is $2 \mathrm{~ms}$ and its will up to $8 \mathrm{~ms}$ and down back to $2 \mathrm{~ms}$. There is a high peak in $58 \mathrm{~ms}$ time taken where it might be the text is quite long to match. The Id row 24 shows the result that has accuracy $1 \%$ or 0.1 probability matched and 58 milliseconds time taken. In these stages, time taken is too high because some each time taken depends on size/length of text or characters to be searched in a list.

By referring to this achievements, the rules state or initiate the achieve level depends on the variable of accuracy and time taken. It can be seen that the achievement for pattern that match in Id 16, is in achieve level rank number 5 while Id 13,14 and 15 is 1,2 and 3 rank although the accuracy and time taken is does not have a tendency to high.

Table 5.1 Accuracy, Time Taken and Achievement for Pattern Searching

\begin{tabular}{|c|c|c|c|c|}
\hline Id & ISBN Code & $\begin{array}{l}\text { Accuracy } \\
\text { (percentage) }\end{array}$ & $\begin{array}{c}\text { Time } \\
\text { Taken(ms) }\end{array}$ & $\begin{array}{c}\text { Achieve } \\
\text { ment }\end{array}$ \\
\hline 1 & ISBN-20001108 & $0 \%$ & 2 & 3 \\
\hline 2 & ISBN-20001109 & $0 \%$ & 2 & 7 \\
\hline 3 & ISBN-20001130 & $18 \%$ & 2 & 14 \\
\hline 4 & ISBN-20001118 & $0 \%$ & 2 & 15 \\
\hline 5 & ISBN-20001101 & $0 \%$ & 2 & 16 \\
\hline 6 & ISBN-20001107 & $0 \%$ & 2 & 17 \\
\hline 7 & ISBN-20001106 & $6 \%$ & 2 & 20 \\
\hline 8 & ISBN-20001105 & $0 \%$ & 2 & 21 \\
\hline 9 & ISBN-20001104 & $0 \%$ & 2 & 28 \\
\hline 10 & ISBN-20001103 & $0 \%$ & 2 & 29 \\
\hline 11 & ISBN-20001102 & $0 \%$ & 2 & 30 \\
\hline 12 & ISBN-20001131 & $0 \%$ & 2 & 24 \\
\hline 13 & ISBN-20001132 & $0 \%$ & 2 & 1 \\
\hline 14 & ISBN-20001133 & $18 \%$ & 2 & 2 \\
\hline 15 & ISBN-20001134 & $5 \%$ & 2 & 4 \\
\hline 16 & ISBN-20001135 & $100 \%$ & 2 & 5 \\
\hline 17 & ISBN-20001136 & $12 \%$ & 2 & 6 \\
\hline 18 & ISBN-20001137 & $0 \%$ & 2 & 8 \\
\hline 19 & ISBN-20001138 & $0 \%$ & 8 & 9 \\
\hline 20 & ISBN-20001139 & $14 \%$ & 2 & 10 \\
\hline 21 & ISBN-20001370 & $11 \%$ & 4 & 11 \\
\hline 22 & ISBN-20001772 & $0 \%$ & 2 & 12 \\
\hline 23 & ISBN-20001773 & $0 \%$ & 2 & 13 \\
\hline 24 & ISBN-20001774 & $1 \%$ & 58 & 18 \\
\hline 25 & ISBN-20001775 & $0 \%$ & 2 & 19 \\
\hline 26 & ISBN-20001776 & $0 \%$ & 2 & 22 \\
\hline 27 & ISBN-20001777 & $0 \%$ & 2 & 23 \\
\hline 28 & ISBN-20001778 & $3 \%$ & 2 & 25 \\
\hline 29 & ISBN-20001779 & $6 \%$ & 2 & 26 \\
\hline 30 & ISBN-20001771 & $7 \%$ & 2 & 27 \\
\hline
\end{tabular}

\section{SUMMARY AND CONCLUSION}

This chapter presents detailed summary of the research that have been conducted including the methods use, the obtained outcomes and major conclusion arising from work. It also states the contribution of the research and suggests the future work that can be made to improve the research in the future.

\subsection{Summary of the Research}

This research has demonstrated that when the QR Image is scanned by Mobile Application, the QR is successfully decoded to text as ID and tracked the information from server. The SDK for QR code is reliable for the system application for mobile device because it has a recognition item where user can move up and down for any dimension. The QR code also has the capability to read at 360 degree rotation sensor/camera from mobile phone based on plot recognition.

The string matching is an automatic recognition of the text of string by referring to the pattern of text which uses variety of technique to recognize the text. It also can search each of the character faster. The text is recognized by pattern based on the methodology to build the string matching on this system. Boyer Moore technique for text searching for library book information is among the best way because it can check the pattern of text. Moreover, the system is developed by using java code which applicable and compatible in any platform of current technology or android development. Each successful of the searching is representing to the accuracy of the text.

Furthermore, each single character processing for every single text is measured. The text is measured according to the time to determine the processing of time taken to finish in checking the text based on pattern of text. The faster the value of the time, the more efficient the system is. Boyer Moore and rule based system look like as an engine to calculate the accuracy of the text based on time taken to process and generate the best result. This approach is executed in real time and robust string matching to be more accurate and become faster with respects to variation in length and pattern of text.

It can be found that the result of implementation artifacts has been tested that hybrid of Boyer Moore and rule based system algorithms for the particular string matching is proved and useful in all these application. Thus, by hybrid of Boyer Moore and rule based system for book information is fulfilling the approach.

\subsection{Conclusion}

By referring to the previous chapters, the proposed model has achieved the objectives of this study. The hybrid approach of Boyer Moore and rule based system method produce better accuracy for string matching. Besides, the time taken performance has shown result when being applied to small data set. However, its performance time taken depends on the size of the text increased.

\section{ACKNOWLEDGMENTS}

This paper is part of Master of Computer Science (Software Engineering and Intelligence) research work in the Faculty of Information and Communication Technology, UTeM

\section{REFERENCES}

[1] A Abdullah \& A.N. Zainab (2007), Collaborative Digital Library of Historical Resources: Evaluation of First Users, Malaysian Journal of Library \& Information Science, Vol. 12, no. 2, pp. 99-122.

[2] K.Kishoreanthuvan, S.Balaji, S.Thamizharasan \& K.Kuzhandaivelu (2013), An Intermediate Service 
Composer Architecture for Dynamic Discovery and Invocation of Web Services from Mobile Devices, IOSR Journal of Computer Engineering (IOSR-JCE), Volume 15, Issue 6 (Nov. - Dec. 2013), pp 45-48.

[3] M. A. S. Naser (2012), Quick-Skip Search Hybrid Algorithm for the Exact String Matching Problem, AlMansour Journal, No.17, Special Issue, pp 119-134.

[4] V. SaiKrishna, A. Rasool \& N. Khare (2012), String Matching and its Applications in Diversified Fields, IJCSI International Journal of Computer Science Issues, Vol. 9, Issue 1, No 1, pp 219-226.

[5] KR Barker, E Attridge, J Bennett and et. al. (2012), The Implementation of Embedded Quick Response Codes into Library Resources to Improve Service Delivery, Journal of the Medical Library Association, 100(1): pp $68-71$
[6] ASH Basari, AM Zain, NK Ibrahim, N Yusof, SA Asmai (2010), A Mobile Disaster Alert Intelligent System, Proceedings of MUCET, Melaka, pp 291-294.

[7] N. Singla \& D.Garg (2012), String Matching Algorithms and their Applicability in various Applications, International Journal of Soft Computing and Engineering (IJSCE) Volume-I, Issue-6, January 2012, pp 218-222.

[8] C. Porcel, A. Tejeda-Lorente, M.A. Martínez \& E. Herrera-Viedma (2012), A hybrid recommender system for the selective dissemination of research resources in a Technology Transfer Office, Information Sciences, Volume 184, Issue 1, pp 1-19.

[9] B. Neelakandan, S. Duraisekar, R.Balasubramani (2010), Implementation of Automated Library Management System in the School of Chemistry Bharathidasan University using Koha Open Source Software. International Journal of Applied Engineering Research, Volume : 1, Issue : 2, pp. 149-167 